\title{
Prestaciones sismorresistentes de un edificio sanitario proyectado con la NCSE-O2
}

\section{Seismic performance of a health facility designed with Spanish NCSE-O2 code}

\author{
F. J. Pérez $^{(*)}$, L. Morillas ${ }^{(*)}$
}

RESUMEN

Se evalúan las prestaciones sismorresistentes de un edificio de uso sanitario proyectado con la normativa española actual. La evaluación ha sido realizada en el marco de una metodología probabilística que provee una base consistente con las incertidumbres del proceso de evaluación. Un prototipo de edificio sanitario sirve como base para discutir una estimación realista de las implicaciones de terremotos raros y ocasionales en edificios de importancia especial. Para este fin, el estudio comprende un análisis dinámico no lineal de la estructura del edificio, la identificación de grupos de prestaciones estructurales y no estructurales, la expresión probabilística de la respuesta de la estructura y la cuantificación de daños y costes de reparación mediante relaciones de fragilidad. Este trabajo revela que las prestaciones sismorresistentes del edificio analizado son inapropiadas en términos de daños, pérdida de funcionalidad y costes de reparación.

Palabras clave: Evaluación sismorresistente; terremotos; instalaciones sanitarias; estructuras porticadas; daños; pérdidas.

\section{ABSTRACT}

We assess the seismic performance of a hospital building designed following current Spanish codes. The assessment is done with a state-of-the-art probabilistic framework which provides a consistent account for the uncertainties in the evaluation process. A prototype of a health facility serves as a basis to discuss a realistic estimate of the implications of occasional and rare ground motion in essential facilities. To this end, this study comprises nonlinear time history analyses of the building structure, the identification of structural and non-structural performance groups, the probabilistic expression of structural response, and the quantification of damage and repair costs by means of fragility relations. This work reveals that the seismic performance of the analysed facility is inappropriate in terms of damage, loss of functionality and repair costs.

Keywords: seismic performance; earthquakes; health facility; frame structures; damage; losses.

(*) Universidad de Valladolid (España).

Persona de contacto/Corresponding author: fcojavierperezjimenez@gmail.com (F. J. Pérez)

ORCID: http://orcid.org/oooo-0oo2-3024-8280 (F. J. Pérez); http://orcid.org/oooo-0oo2-0861-2381 (L. Morillas)

Cómo citar este artículo/Citation: F. J. Pérez; L. Morillas (2018). Prestaciones sismorresistentes de un edificio sanitario proyectado con la NCSE-02. Informes de la Construcción, 70(551): e266. https://doi.org/10.3989/ic.16.117

Copyright: (C) $\mathbf{2 0 1 8}$ CSIC. Este es un artículo de acceso abierto distribuido bajo los términos de la licencia de uso y distribución Creative Commons Reconocimiento 4.0 Internacional (CC BY 4.0). 


\section{INTRODUCCIÓN}

Los hospitales y centros de salud necesitan mostrar un mejor desempeño sísmico que los edificios convencionales debido a que su seguridad es vital tras un evento sísmico. Esta cuestión se aborda en los códigos mediante la amplificación de las fuerzas sísmicas por un factor de importancia. El factor de importancia es un valor empírico cuya lógica es que, si la estructura del edificio puede resistir mayores fuerzas laterales, el daño será menor. Para los edificios de uso sanitario, este valor oscila entre 1,3 en la NCSE-O2 (1), a 1,4 en el Eurocódigo 8 (2), o 1,5 en ASCE 7 (3).

Las fuerzas sísmicas de cálculo son posteriormente divididas por un factor de reducción R (o $\mu$ en NCSE-02) si el sistema de resistencia lateral cumple ciertos requisitos de ductilidad. Debido a que los valores del factor de importancia son menores que el factor de reducción, la aplicación simultánea de ambos significa que la respuesta estructural probablemente sea inelástica en caso del terremoto de proyecto.

Independientemente de si la respuesta estructural es inelástica o no, los daños en los elementos no estructurales, sistemas secundarios y contenidos en los centros sanitarios se estiman entre el 25-75\% de los costes totales de reparación, y han sido la causa principal para la evacuación y la interrupción del uso en instalaciones críticas (4-8). El papel de los elementos no estructurales tradicionalmente no ha sido tenido en cuenta, pero la experiencia ha demostrado que estos elementos tienen un impacto en el comportamiento sísmico de edificios. Los elementos no estructurales y contenidos en el edificio típicamente representan la mayor parte del costo de la construcción, hasta el $92 \%$ en los hospitales (9).

El hecho de que los fallos en elementos no estructurales representen la mayoría de los daños causados por un terremoto en los eventos recientes está siendo reconocido por los códigos. El Eurocódigo 8 (2) establece unos límites de deformación para los niveles de prestaciones de ocupación inmediata (IO), seguridad para la vida (LS), y los niveles de colapso (C). También destaca que el daño no estructural debe ser económicamente reparable después de un terremoto poco frecuente. El Eurocódigo 8 proporciona herramientas para calcular fuerzas estáticas equivalentes en elementos no estructurales, y las demandas de desplazamiento relativo se pueden obtener de los límites de deformación (10). Además, desde la revisión en 2010 de la ASCE 7 (3), se dedica el capítulo 13 al proyecto sismorresistente de los elementos no estructurales. Este capítulo de la ASCE 7 desarrolla cómo estimar la demanda ante un terremoto y especifica requisitos especiales que pretenden mejorar las prestaciones sísmicas. Además, la ASCE 7-10 requiere que algunos sistemas críticos sean sísmicamente calificados por protocolos de prueba $(11,12)$. Asimismo, los nuevos códigos reconocen la importancia de los terremotos menores mediante la introducción de una serie de niveles de prestaciones definidos dentro de rangos aceptables de deformación y demandas de resistencia.

La guía FEMA E-74 (13) ha aumentado el nivel de conciencia sobre los riesgos no estructurales, las costosas consecuencias de los fallos y las oportunidades para mitigar el daño. Para un edificio típico, con un nivel moderado de sacudida como en España, el papel de los elementos no estructurales es tal que el Apéndice E del FEMA E-74 (13) contempla riesgos entre moderados a altos para la seguridad de la vida, daños en elementos y la pérdida funcional. Para un caso análogo al español, la guía sugiere el refuerzo sísmico para la mayoría de los elementos arquitectónicos, componentes eléctricos, mecánicos, tuberías, conductos, artefactos de iluminación y ascensores. Los muebles, accesorios y equipos también están en riesgo y pueden requerir anclaje y arriostramiento.

Con respecto a los elementos no estructurales, la NCSE-O2 contiene algunas disposiciones, y otras regulaciones europeas (14-17) no son de obligado cumplimiento. Como resultado, es probable que las instalaciones sanitarias en las regiones de actividad sísmica de España carezcan de unas prestaciones sismorresistentes adecuadas, ya sea para elementos estructurales, no estructurales, contenidos y equipamiento sanitario en el edificio.

Como las prestaciones sísmicas de edificios sanitarios han sido poco satisfactorias en el pasado (11,18-20), las nuevas investigaciones se centran en una mejor caracterización y evaluación de la fragilidad. Ensayos en mesa sísmica de habitaciones de hospitales han demostrado que el mecanismo oscilante de las cabinas médicas comienza con valores bajos de la aceleración de planta que van desde 0,37-0,61g, lo que lleva al vuelco en 1,og. Del mismo modo, el deslizamiento del mobiliario de oficina del hospital se produce a $0,5 \mathrm{~g}$ (21). Otras pruebas describen fuertes oscilaciones de aparatos médicos, causando la colisión y serio desorden en las salas de operación (22-23). Los contenidos en los estantes comienzan a caer con aceleraciones entre 0,35-0,70g (24). El equipamiento médico que se encuentra sin anclar, como refrigeradores, armarios, incubadoras, goteros de pie, etc. es especialmente sensible (21,25). Desde VISION 2000 (26), la evaluación y el proyecto basado en prestaciones se han convertido en estrategias comunes. Las metodologías basadas en prestaciones (27-29) permiten la consideración de otros criterios de proyecto distintos al de fuerza y las especificaciones de diseño estructural. Un objetivo común para el proyecto sismorresistente es mitigar los daños en los elementos no estructurales del edificio por razones sociales, económicas y de durabilidad. El proyecto ATC-58 (29) ha implementado enfoques sistemáticos para la evaluación de daños en edificios nuevos y existentes, y de la incertidumbre en el rendimiento probable del edificio.

\section{METOdOLOGÍA DE LA ATC-58 PARA EVALUACIÓN SISMORRESISTENTE BASADA EN PRESTACIONES}

La evaluación de las prestaciones del edificio en este artículo sigue la metodología de la ATC-58 (29). La base técnica de la metodología es el marco para la ingeniería sísmica basada en prestaciones desarrollado por investigadores del Pacific Earthquake Research Center (PEER) entre 1997 y 2010 (30). Esencialmente, se aplica el teorema de la probabilidad total para predecir las consecuencias del terremoto en términos de la probabilidad de alcanzar valores particulares de las medidas de prestaciones. El marco se divide en los cuatro pasos de la Fig. 1.

El primer paso implica un análisis de peligrosidad sísmica, a través del cual se evalúan una o más medidas de intensidad de movimiento del suelo (IM) para un determinado emplazamiento. El análisis de peligrosidad tiene como finalidad la caracterización de la acción sísmica para el análisis de la respuesta del edificio. Las medidas de la intensidad del terremo- 


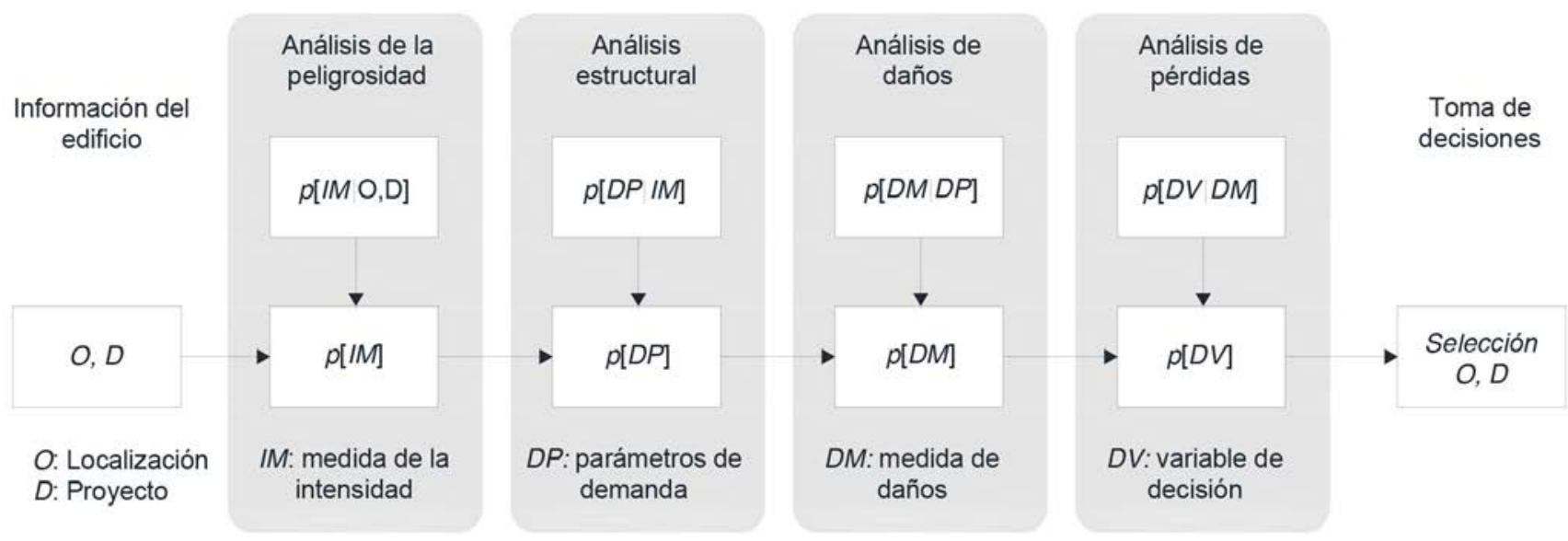

Figura 1. Metodología de evaluación de la ATC 58.

to (IM) pueden obtenerse a través de mapas de peligrosidad o de otros análisis probabilistas del peligro sísmico.

Para unas IM dadas y los correspondientes movimientos del suelo, el segundo paso es realizar simulaciones para el cálculo de parámetros de la demanda (DP), que caracterizan la respuesta en términos de deformaciones, aceleraciones, fuerzas, disipación de energía, etc. En los edificios, los DP más comunes son los valores de desplazamiento entre plantas, deformaciones inelásticas y tensiones, y las aceleraciones de planta. Las relaciones entre DP e IM se pueden obtener a través de los análisis dinámicos no lineales. El resultado del análisis estructural caracteriza la probabilidad condicional $\mathrm{p}(\mathrm{DP} \mid \mathrm{IM})$ de exceder un determinado valor de DP para una IM dada.

El siguiente paso en el proceso es llevar a cabo un análisis de daños, que relaciona los DP con las medidas de daño (DM), que describen los daños que sufre el edificio o instalación. Las DM incluyen descripciones de los daños en las partes estructurales, no estructurales, componentes y contenidos, con el fin de cuantificar las reparaciones necesarias, y las implicaciones de seguridad funcional o de la vida de sus ocupantes. Suelen utilizarse curvas de fragilidad (CF) para estimar la probabilidad de que se alcance un determinado estado de daños (DS) para un valor dado de un DP. Las curvas de fragilidad son funciones estadísticas que representan la probabilidad de que un estado de daño se haya alcanzado o superado, dada una medida cuantitativa del parámetro de la demanda (DP).

El paso final en la evaluación es calcular las variables de decisión (DV) en términos que sean significativos, por ejemplo: las pérdidas monetarias directas, el tiempo de restauración y las bajas. Las DV se determinan mediante la traducción de los estados de daño de los elementos del edificio en coste o tiempo de restauración.

\section{CARACTERIZACIÓN DE LA ACCIÓN SÍSMICA}

Debido a que los efectos de los terremotos menores en edificios modernos han superado nuestras expectativas, los códigos de todo el mundo exigen ahora que el comportamiento sísmico se compruebe con al menos dos niveles de intensidad. El Eurocódigo 8 (2) se refiere a periodos de retorno $T_{R}=475 \mathrm{y}$ $T_{R}=95$ años para los terremotos raros y ocasionales. Esto se corresponde con las probabilidades de excedencia de $10 \%$ en 50 años, y $10 \%$ en 10 años.
El análisis de la peligrosidad se basa en estos dos niveles de intensidad en el emplazamiento del edificio: Granada, España. La NCSE-O2 (1) proporciona directamente el espectro elástico de respuesta para terremotos raros, que se muestra en línea gruesa en la Fig. 2a para una aceleración básica horizontal $a_{b}=0,23 g$ y terreno tipo III. El espectro de respuesta para el terremoto ocasional en la Fig. 2b se obtiene considerando la expresión aproximada de la NCSE-O2 (1) para estimar la aceleración horizontal correspondiente a un periodo de retorno (TR) diferente al de 500 años, multiplicando por $[T R / 500]^{0.4}$.

Se han seleccionado dos conjuntos de cinco registros históricos europeos que cumplen los siguientes criterios: (i) el valor promedio de las ondas de cizalla en los primeros $30 \mathrm{~m}$ del terreno $\left(\mathrm{V}_{\mathrm{s} 30}\right)$ esté entre 200 y $400 \mathrm{~m} / \mathrm{s}$, (ii) la magnitud de momento $\mathrm{M}_{\mathrm{w}}^{\mathrm{s} 30}>5.5$ para sismos raros, (iii) la magnitud de momento $\mathrm{M}_{\mathrm{w}}<5.5$ para sismos ocasionales, (iv) duración mínima de 10s. Los principales parámetros de los acelerogramas originales se recogen en la Tabla 1. Estos conjuntos de acelerogramas se ajustan a los espectros de respuesta de la NCSE-02 usando el algoritmo de wavelets propuesto por Abrahamson (31) y revisado por Hancock et al. (32). En el escalado de acelerogramas, se observan los criterios del Eurocódigo 8 y la NCSE-02. En la Fig. 2 se muestra la media de los valores de la respuesta espectral de los acelerogramas con línea roja.

\section{PROTOTIPO DE UN EDIFICIO DE USO SANITARIO}

Para realizar la evaluación sismorresistente basada en prestaciones de una construcción hospitalaria en una zona de sismicidad moderada, se analiza un prototipo representativo. El esquema del edificio es de bloques lineales y paralelos de forma que se asemeja a construcciones recientes de uso hospitalario. Consta de planta baja +2 plantas, siendo la altura de las plantas tipo de $3,10 \mathrm{~m}$, mientras que la altura de planta baja es de 3,30m. La planta baja se dedica a administración y consultas, mientras que el resto son habitaciones de estancia hospitalaria.

\subsection{Proyecto y modelo de la estructura}

Se analiza un modelo numérico del pórtico exterior Y8 en la Fig. 3. La estructura del edificio se proyecta según el método 
Tabla 1. Características de los acelerogramas originales.

\begin{tabular}{|c|c|c|c|c|c|c|}
\hline Registro & Nombre estación & $\begin{array}{c}\text { Fecha } \\
\text { DD/MM/AAAA }\end{array}$ & $\begin{array}{l}\text { Distancia } \\
(\mathbf{k m})\end{array}$ & $\begin{array}{l}\text { Magnitud } \\
\left(M_{w}\right)\end{array}$ & $\mathrm{V}_{\mathrm{s} 3 \mathrm{o}}(\mathrm{m} / \mathrm{s})$ & PGA (g) \\
\hline TK.9907 & AI_159_FI & $12 / 11 / 1999$ & 32,3 & 7,0 & 316 & 0,25 \\
\hline TK.8101 & AI_011_DZC & 17/08/1999 & 101,2 & 7,4 & 282 & 0,37 \\
\hline TK.0302 & AI_137_DIN & 01/10/1995 & 0,5 & 6,4 & 198 & 0,32 \\
\hline TK.0105 & AI_O22_CYH_TIM & 27/06/1998 & 48,2 & 6,2 & 263 & 0,22 \\
\hline TK.1401 & AI_O10_BOL & $12 / 11 / 1999$ & 36,1 & 7,3 & 294 & 0,80 \\
\hline IT.SRP & Sorbolo (Pezzani) & $27 / 01 / 2012$ & 50,1 & 5,0 & 218 & 0,07 \\
\hline IT.BOJ & Bojano (Nuova) & $29 / 12 / 2013$ & 10,4 & 5,0 & 306 & 0,04 \\
\hline TK.5402 & AI_oo6_AKY & $23 / 08 / 2000$ & 17,0 & 5,2 & 271 & 0,09 \\
\hline IT.MAA & Majano - Ascensore & 17/06/1976 & 14,3 & 5,2 & 344 & 0,07 \\
\hline TK.1606 & AI_204_BYTo4 & $24 / 10 / 2006$ & 14,5 & 4,8 & 301 & 0,18 \\
\hline
\end{tabular}

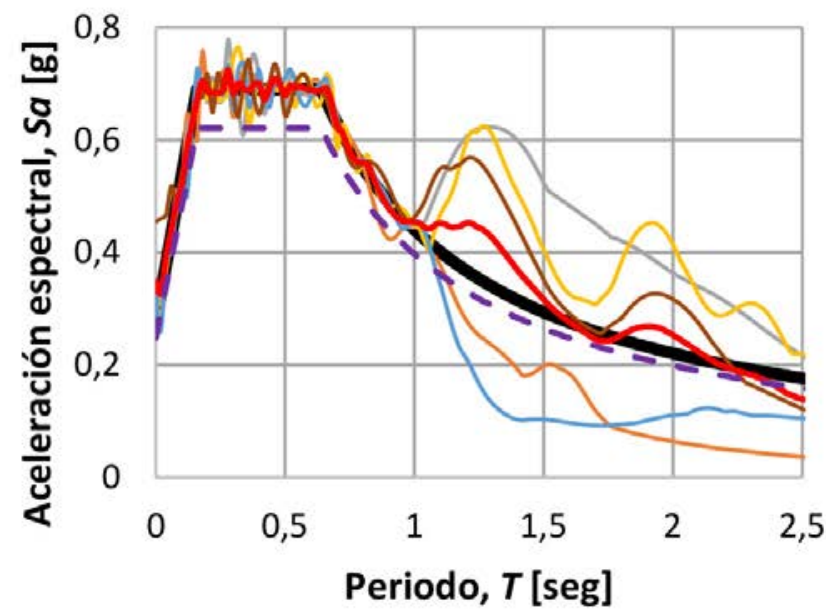

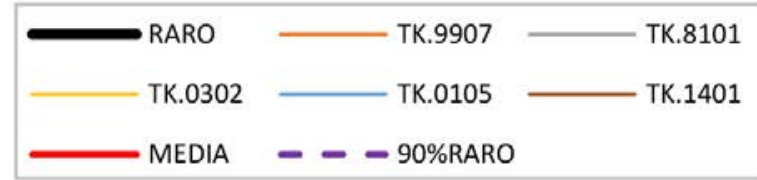

(a)
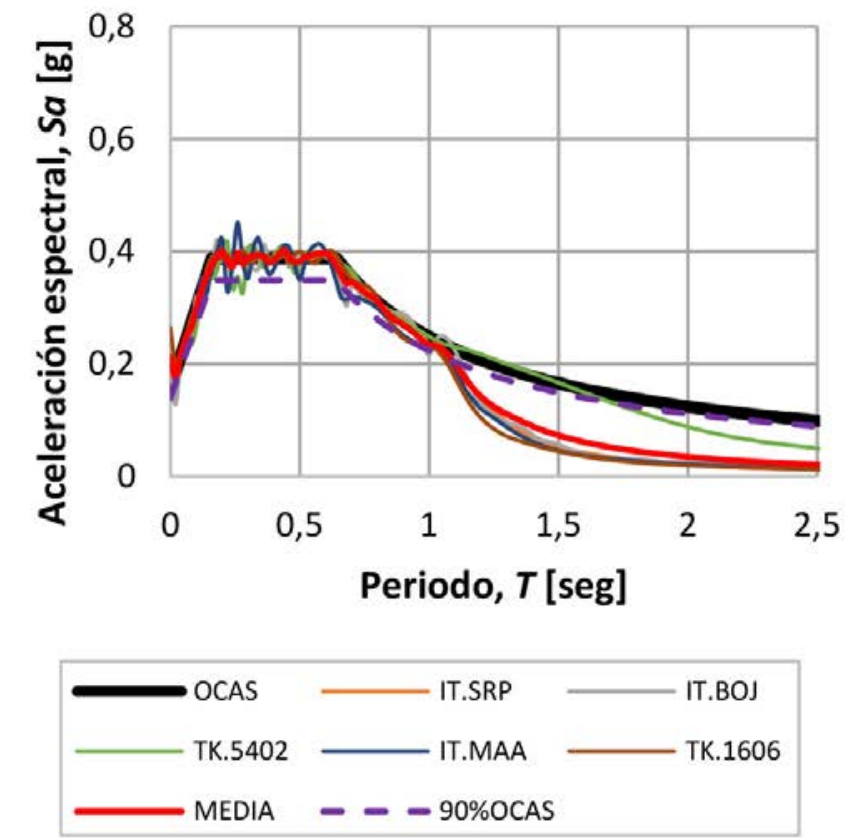

(b)

Figura 2. Espectros de respuesta. (a) Sismo raro. (b) Sismo ocasional.

de los estados límite siguiendo los criterios de la normativa española actual $(1,33,34)$ considerando que es de importancia especial. Está formada por una retícula de 7x3 pórticos de hormigón armado con luces de $5 \mathrm{~m}$ en ambas direcciones, con forjados unidireccionales $25+5$ y vigas de canto. El cálculo de la estructura del módulo seleccionado del edificio ha sido realizado con CYPECAD (35). Los valores característicos de las acciones se han tomado del CTE-DB-SE-AE (33): peso específico del hormigón armado $25 \mathrm{kN} / \mathrm{m}^{3}$, peso propio del forjado $4 \mathrm{kN} / \mathrm{m}^{2}$, pavimento $1 \mathrm{kN} / \mathrm{m}^{2}$, tabiquería $1 \mathrm{kN} / \mathrm{m}^{2}$, cubierta plana $2.5 \mathrm{kN} / \mathrm{m}^{2}$, cerramientos $8 \mathrm{kN} / \mathrm{m}$, sobrecarga de uso $2 \mathrm{kN} / \mathrm{m}^{2}$.

La estructura ha sido proyectada para resistir un terremoto de proyecto caracterizado por un espectro de respuesta, considerando un factor de importancia $\rho=1,3$, tipo de suelo III (velocidad de propagación de las ondas transversales $\mathrm{V}_{\mathrm{s} 30}$ entre 200 y $400 \mathrm{~m} / \mathrm{s}$ ), factor de reducción de fuerzas sísmicas por ductilidad $\mu=3$ y una aceleración básica de 0,23g. La resistencia característica a compresión del hormigón es $\mathrm{f}_{\mathrm{ck}}=25 \mathrm{MPa}$, el límite elástico del acero de armar es $\mathrm{f}_{\mathrm{yk}}=500 \mathrm{MPa}$. La sección transversal de los pilares es de $40 \mathrm{x} 40 \mathrm{~cm}^{2}$. La sección de las vigas principales en la dirección del eje $Y$ es de $40 \times 50 \mathrm{~cm}^{2}$, y la de las vigas perpendiculares en la dirección del eje X es de 40 x $40 \mathrm{~cm}^{2}$. En la Tabla 2 se detallan las dimensiones y el armado de vigas y pilares del pórtico Y8 que es representativo de la estructura. El periodo fundamental del pórtico es $T_{1}=0,52 \mathrm{~s}$, con un $89,4 \%$ de masa modal. Los siguientes periodos de vibración son $T_{2}=0,17 \mathrm{~s}$ y $T_{3}=0,10 \mathrm{~s}$, con un $9 \%$ y un $1,6 \%$ de masa modal respectivamente. El coeficiente cortante basal del pórtico es $\alpha=0,30$.

La estructura porticada de hormigón armado ha sido modelada en 2D con SeismoStruct (36) para realizar cálculos dinámicos directos y obtener su respuesta no lineal a terremotos. Las secciones trasversales se modelaron como secciones rectangulares de hormigón armado con la geometría y el armado correspondiente al prototipo. El modelo constitutivo para el hormigón es el propuesto por Mander (con_ma), con resistencia característica a compresión de $25 \mathrm{MPa}$, resistencia característica a tracción 2,2 MPa, y módulo de elasticidad 


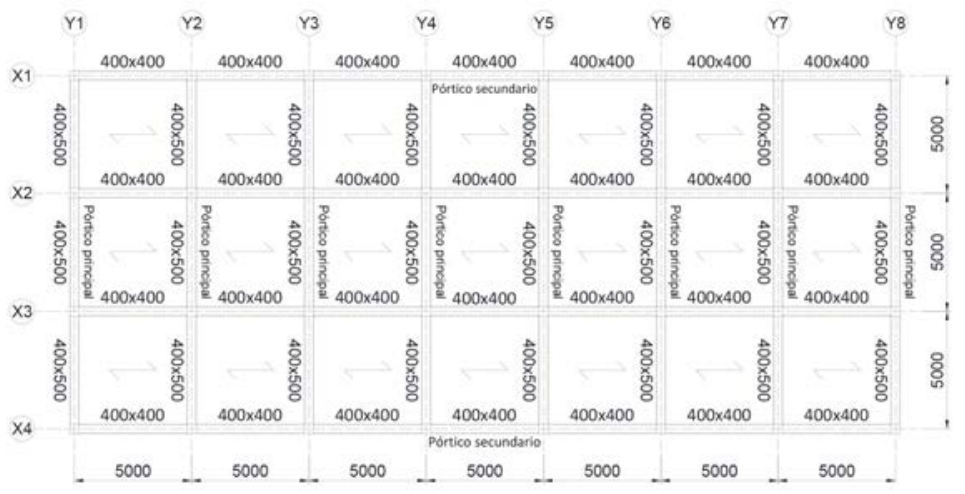

(a) Planta.

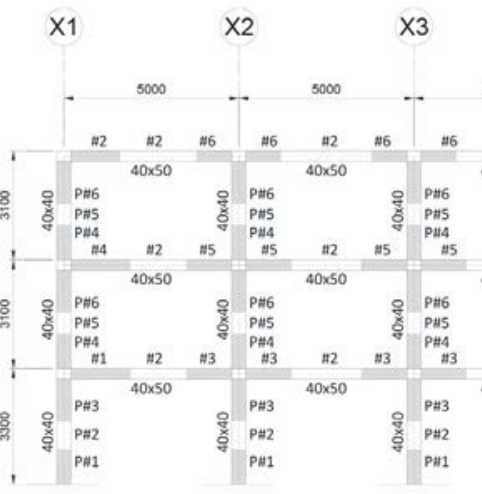

(b) Alzado del pórtico Y8.

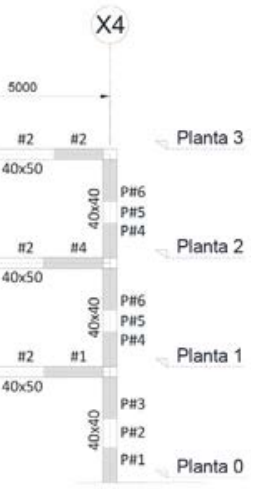

Figura 3. Estructura del edificio hospitalario.

Tabla 2. Descripción de las dimensiones y el armado de las vigas y pilares del pórtico 8 .

\begin{tabular}{|c|c|c|c|c|c|c|c|}
\hline \multicolumn{8}{|c|}{ Armado de vigas del Pórtico 8} \\
\hline \multirow{2}{*}{$\begin{array}{l}\text { Tipo de } \\
\text { sección }\end{array}$} & \multicolumn{2}{|c|}{ Dimensiones } & \multicolumn{3}{|c|}{ Barras } & \multicolumn{2}{|c|}{ Estribos } \\
\hline & $\operatorname{Ancho}(X)$ & Alto (Y) & $\begin{array}{l}\text { Armadura } \\
\text { superior }\end{array}$ & $\begin{array}{l}\text { Armadura } \\
\text { inferior }\end{array}$ & $\begin{array}{c}\text { Armadura } \\
\text { de piel }\end{array}$ & Diámetro & $\begin{array}{c}\text { Separación } \\
(\mathrm{cm})\end{array}$ \\
\hline$\# 1$ & 40 & 50 & $6 \varnothing_{16}, 3 \varnothing_{12}$ & $4 \varnothing_{20}$ & $2 Ø 10$ & $1 \varnothing 8$ & 10 \\
\hline$\# 2$ & 40 & 50 & $4 \varnothing 16$ & $4 \varnothing 20$ & $2 \varnothing 10$ & $1 \varnothing 8$ & 18 \\
\hline$\# 3$ & 40 & 50 & $7 \varnothing_{16,2020}$ & $4 \varnothing_{20}$ & $2 \varnothing 10$ & $1 \varnothing 8$ & 10 \\
\hline$\# 4$ & 40 & 50 & $4 \varnothing_{16,4 \varnothing_{12}}$ & $3 \varnothing 20$ & $2 \varnothing 10$ & $1 \varnothing 8$ & 10 \\
\hline$\# 5$ & 40 & 50 & $9 \varnothing_{16}$ & $3 \varnothing_{20}$ & $2 \varnothing 10$ & $1 \varnothing 8$ & 10 \\
\hline$\# 6$ & 40 & 50 & $7 \varnothing 16$ & $3 \varnothing 20$ & $2 \varnothing 10$ & $1 \varnothing 8$ & 10 \\
\hline \multicolumn{8}{|c|}{ Armado de pilares del Pórtico 8} \\
\hline \multirow{2}{*}{$\begin{array}{l}\text { Tipo de } \\
\text { sección }\end{array}$} & \multicolumn{2}{|c|}{ Dimensiones } & \multicolumn{3}{|c|}{ Barras } & \multicolumn{2}{|c|}{ Estribos } \\
\hline & $\operatorname{Ancho}(X)$ & Alto (Y) & Esquina & Cara $(X)$ & $\operatorname{Cara}(Y)$ & Diámetro & $\begin{array}{c}\text { Separación } \\
\text { (cm) }\end{array}$ \\
\hline $\mathrm{P} \# 1$ & 40 & 40 & $4 \varnothing_{20}$ & $6 \varnothing_{12}$ & $6 \varnothing 12$ & $1 \varnothing 8$ & 6 \\
\hline $\mathrm{P} \# 2$ & 40 & 40 & $4 \varnothing_{20}$ & $6 \varnothing_{12}$ & $6 \varnothing 12$ & $1 \varnothing 8$ & 15 \\
\hline $\mathrm{P} \# 3$ & 40 & 40 & $4 \varnothing_{20}$ & $6 \varnothing_{12}$ & $6 \varnothing 12$ & $1 \varnothing 8$ & 8 \\
\hline $\mathrm{P} \# 4$ & 40 & 40 & $4 \varnothing 16$ & $4 \varnothing_{12}$ & $4 \varnothing 12$ & $1 \varnothing 8$ & 6 \\
\hline $\mathrm{P} \# 5$ & 40 & 40 & $4 \varnothing 16$ & $4 \varnothing_{12}$ & $4 \varnothing 12$ & $1 \varnothing 8$ & 15 \\
\hline $\mathrm{P} \# 6$ & 40 & 40 & $4 \varnothing_{16}$ & $4 \varnothing_{12}$ & $4 \varnothing_{12}$ & $1 \varnothing 8$ & 8 \\
\hline
\end{tabular}

$23500 \mathrm{MPa}$. Para las barras de refuerzo de acero, se usa la ley constitutiva de Menegotto-Pinto (stp_mp), con módulo de elasticidad $200 \mathrm{GPa}$, límite elástico $500 \mathrm{MPa}$, y parámetro de endurecimiento por deformación $\mu=0,005$. El factor de confinamiento se calcula en base al refuerzo de la sección. Se ha utilizado el modelo de amortiguamiento viscoso de Rayleigh, que es proporcional a una combinación lineal de la masa y la rigidez. Se ha considerado un amortiguamiento del $5 \%$ para el primer y tercer modo de vibración traslacional del pórtico.

\subsection{Grupos de prestaciones y curvas de fragilidad}

Los elementos del edificio se clasifican en 59 grupos de prestaciones (GP) como se muestra en la Tabla 3. Cada grupo de prestaciones es un conjunto de elementos estructurales o no estructurales, cuyo comportamiento está afectado de manera similar por un cierto parámetro de demanda (DP): sufren daños y son sensibles a las pérdidas de manera similar. La probabilidad de que un componente de un determinado GP resulte dañado está estrechamente ligada a un único parámetro de la demanda tal como el desplome o deriva entre plantas $d u_{i}$, o la aceleración de planta $a_{i}$, en la correspondiente planta $i$ del edificio. El nivel o corresponde al suelo de planta baja, y el nivel 3 es la cubierta del edificio. Los daños en la estructura del edificio, cerramientos, elementos no estructurales (particiones, puertas, acristalamientos) son considerados sensibles a la deriva entre plantas, mientras que la aceleración de planta puede dañar otros elementos no estructurales (luces, rociadores, sistemas de alarma de incendios, equipos de climatización en cubierta, equipamiento médico y de enfermería, así como sistemas informáticos). Por ejemplo, el GP58 está compuesto por los equipos médicos sensibles a la aceleración del suelo de planta primera $a_{1}$.

La probabilidad de que cierto elemento de un edificio sufra daños en función de un DP viene dado por curvas de fragilidad (CF). Las curvas de fragilidad son funciones estadísticas que representan la probabilidad de que no se exceda un determinado estado de daño (DS) dada una medida cuantitativa de un parámetro de demanda (DP). Las curvas de fragilidad suelen expresarse como funciones de distribución lognormal acumulada como en la Fig. 4. Estas funciones se definen con el logaritmo natural de los valores medios $(\lambda)$ en los que hay un $50 \%$ de probabilidad de que se inicie un cierto nivel de daño 
Tabla 3. Elementos en los grupos de prestaciones y parámetros de curvas de fragilidad.

\begin{tabular}{|c|c|c|c|c|c|c|c|c|c|c|c|}
\hline \multirow{2}{*}{ GP } & \multirow{2}{*}{ Elementos en el grupo } & \multirow{2}{*}{ DP } & \multirow{2}{*}{ Localización } & \multirow{2}{*}{ Coste (€) } & \multirow{2}{*}{$\mathbf{C F}$} & DS1 & DS2 & DS3 & DS1 & DS2 & DS3 \\
\hline & & & & & & $\lambda \mathbf{1}$ & $\lambda 2$ & $\lambda_{3}$ & $\zeta_{1}$ & $\zeta 2$ & $\zeta 3$ \\
\hline 1 & Estructura HA_1 & du1 & Entre niveles o y 1 & 146535 & 1 & $-0,70$ & $-0,35$ & $-0,01$ & 0,05 & 0,05 & 0,05 \\
\hline 2 & Estructura HA_2 & du2 & Entre niveles 1 y 2 & 136068 & 1 & & & & & & \\
\hline 3 & Estructura $\mathrm{HA} \_3$ & du3 & Entre niveles 2 y 3 & 136068 & 1 & & & & & & \\
\hline 4 & Cerramientos_o1 & du1 & Entre niveles o y 1 & 58614 & 2 & $-1,20$ & $-0,40$ & 0,95 & 0,40 & 0,30 & 0,20 \\
\hline 5 & Cerramientos_12 & du2 & Entre niveles 1 y 2 & 54427 & 2 & & & & & & \\
\hline 6 & Cerramientos_23 & du3 & Entre niveles 2 y 3 & 54427 & 2 & & & & & & \\
\hline 7 & Tabiquería_o1 & du1 & Entre niveles o y 1 & 25748 & 3 & $-0,69$ & $-0,19$ & 0,08 & 0,50 & 0,50 & 0,50 \\
\hline 8 & Tabiquería_12 & du2 & Entre niveles 1 y 2 & 23864 & 3 & & & & & & \\
\hline 9 & Tabiquería_23 & du3 & Entre niveles 2 y 3 & 23655 & 3 & & & & & & \\
\hline 10 & Carpinterías_o & du1 & En nivel o & 7117 & 4 & $-2,37$ & $-0,76$ & $-0,07$ & 0,70 & 0,70 & 0,70 \\
\hline 11 & Carpinterías_1 & du2 & En nivel 1 & 6908 & 4 & & & & & & \\
\hline 12 & Carpinterías_2 & du3 & En nivel 2 & 6908 & 4 & & & & & & \\
\hline 13 & Escaleras_o & du1 & En nivel o & 18422 & 5 & $-0,80$ & 0,65 & 1,10 & 0,60 & 0,50 & 0,40 \\
\hline 14 & Escaleras_1 & du2 & En nivel 1 & 17165 & 5 & & & & & & \\
\hline 15 & Escaleras_2 & du3 & En nivel 2 & 16747 & 5 & & & & & & \\
\hline 16 & Falsos techos_1 & a1 & Bajo nivel 1 & 25120 & 6 & $-1,00$ & $-0,60$ & $-0,25$ & 0,46 & 0,40 & 0,50 \\
\hline 17 & Falsos techos_2 & $\mathrm{a} 2$ & Bajo nivel 2 & 24074 & 6 & & & & & & \\
\hline 18 & Falsos techos_3 & a3 & Bajo nivel 3 & 24074 & 6 & & & & & & \\
\hline 19 & Petos_3 & a3 & En nivel 3 & 10467 & 7 & $-1,03$ & $-0,42$ & $-0,14$ & 0,46 & 0,61 & 0,74 \\
\hline 20 & Puertas_o & ao & En nivel o & 56102 & 8 & $-0,85$ & $-0,62$ & 0,25 & 0,44 & 0,56 & 0,75 \\
\hline 21 & Puertas_1 & $\mathrm{a} 1$ & En nivel 1 & 55683 & 8 & & & & & & \\
\hline 22 & Puertas_2 & $\mathrm{a} 2$ & En nivel 2 & 55683 & 8 & & & & & & \\
\hline 23 & Ascensores_o & ao & En nivel o & 29726 & 9 & $-0,60$ & $-0,60$ & $-0,60$ & 0,40 & 0,40 & 0,40 \\
\hline 24 & Ascensores_1 & $\mathrm{a} 1$ & En nivel 1 & 27632 & 9 & & & & & & \\
\hline 25 & Ascensores_2 & $\mathrm{a} 2$ & En nivel 2 & 27632 & 9 & & & & & & \\
\hline 26 & Ascensores_3 & a3 & En nivel 3 & 9211 & 9 & & & & & & \\
\hline 27 & Climatización_3 & a3 & En nivel 3 & 36634 & 10 & 0,40 & 0,85 & 0,85 & 0,40 & 0,40 & 0,40 \\
\hline 28 & Caldera_o & ao & En nivel o & 26167 & 11 & $-0,40$ & $-0,23$ & $-0,23$ & 0,55 & 0,45 & 0,45 \\
\hline 29 & Torre refrigeración_3 & a3 & En nivel 3 & 41867 & 12 & $-0,70$ & $-0,70$ & $-0,70$ & 0,53 & 0,53 & 0,53 \\
\hline 30 & Conductos AC_1 & a1 & Bajo nivel 1 & 51287 & 13 & 0,38 & 0,78 & 0,78 & 0,45 & 0,45 & 0,45 \\
\hline 31 & Conductos AC_2 & a2 & Bajo nivel 2 & 47728 & 13 & & & & & & \\
\hline 32 & Conductos AC_3 & a3 & Bajo nivel 3 & 47519 & 13 & & & & & & \\
\hline 33 & Tuberías_1 & $\mathrm{a} 1$ & Bajo nivel 1 & 42286 & 14 & 0,35 & 1,00 & 1,00 & 0,30 & 0,45 & 0,45 \\
\hline 34 & Tuberías_2 & $\mathrm{a} 2$ & Bajo nivel 2 & 41658 & 14 & & & & & & \\
\hline 35 & Tuberías_3 & a3 & Bajo nivel 3 & 41658 & 14 & & & & & & \\
\hline 36 & Red de rociadores_1 & $\mathrm{a} 1$ & Bajo nivel 1 & 29307 & 15 & $-0,35$ & $-0,06$ & $-0,06$ & 0,55 & 0,55 & 0,55 \\
\hline 37 & Red de rociadores_2 & $\mathrm{a} 2$ & Bajo nivel 2 & 27214 & 15 & & & & & & \\
\hline 38 & Red de rociadores_3 & a3 & Bajo nivel 3 & 27214 & 15 & & & & & & \\
\hline 39 & Sistemas PCI_1 & a1 & Bajo nivel 1 & 29307 & 16 & $-0,58$ & 0,25 & 0,25 & 0,49 & 0,75 & 0,75 \\
\hline 40 & Sistemas PCI_2 & $\mathrm{a} 2$ & Bajo nivel 2 & 27214 & 16 & & & & & & \\
\hline 41 & Sistemas PCI_3 & a3 & Bajo nivel 3 & 27214 & 16 & & & & & & \\
\hline 42 & Alumbrado de emergencia_1 & $\mathrm{a} 1$ & Bajo nivel 1 & 7117 & 17 & $-0,71$ & $-0,62$ & $-0,62$ & 0,32 & 0,33 & 0,33 \\
\hline 43 & Alumbrado de emergencia_2 & $\mathrm{a} 2$ & Bajo nivel 2 & 6908 & 17 & & & & & & \\
\hline 44 & Alumbrado de emergencia_3 & a3 & Bajo nivel 3 & 6908 & 17 & & & & & & \\
\hline 45 & Luminarias_1 & $\mathrm{a} 1$ & Bajo nivel 1 & 20934 & 18 & $-0,78$ & $-0,61$ & $-0,41$ & 0,51 & 0,53 & 0,55 \\
\hline 46 & Luminarias_2 & a2 & Bajo nivel 2 & 20934 & 18 & & & & & & \\
\hline 47 & Luminarias_3 & a3 & Bajo nivel 3 & 20934 & 18 & & & & & & \\
\hline 48 & Aseos, Aparatos sanitarios_o & ao & En el nivel o & 31400 & 19 & $-0,69$ & $-0,53$ & $-0,53$ & 0,44 & 0,40 & 0,40 \\
\hline 49 & Aseos, Aparatos sanitarios_1 & a1 & En el nivel 1 & 31400 & 19 & & & & & & \\
\hline 50 & Aseos, Aparatos sanitarios_2 & $\mathrm{a} 2$ & En el nivel 2 & 10467 & 19 & & & & & & \\
\hline 51 & Sistemas informáticos_o & ao & En el nivel o & 19887 & 20 & $\mathrm{O}, 01$ & 0,01 & 0,01 & 0,52 & 0,52 & 0,52 \\
\hline 52 & Sistemas informáticos_1 & $\mathrm{a} 1$ & En el nivel 1 & 5861 & 20 & & & & & & \\
\hline 53 & Sistemas informáticos_2 & a2 & En el nivel 2 & 5652 & 20 & & & & & & \\
\hline 54 & Equipamiento de oficina_o & ao & En el nivel o & 5233 & 21 & 0,02 & 0,02 & 0,02 & 0,40 & 0,40 & 0,40 \\
\hline 55 & Archivadores_o & ao & En el nivel o & 3140 & 22 & $-0,80$ & 0,20 & 0,20 & 0,30 & 0,20 & 0,20 \\
\hline 56 & Estanterías_o & ao & En el nivel o & 2093 & 23 & $-1,19$ & $-0,88$ & $-0,69$ & 0,39 & 0,35 & 0,43 \\
\hline 57 & Equipamiento médico_o & ao & En el nivel o & 104667 & 24 & $-1,06$ & $-0,95$ & $-0,14$ & 0,46 & 0,50 & 0,74 \\
\hline 58 & Equipamiento médico_1 & $\mathrm{a} 1$ & En el nivel 1 & 83734 & 24 & & & & & & \\
\hline 59 & Equipamiento médico_2 & a2 & En el nivel 2 & 83734 & 24 & & & & & & \\
\hline
\end{tabular}


DS, y con las desviaciones logarítmicas estándar (ろ), que indican la incertidumbre de la ocurrencia de este nivel de daño. En general, el estado de daño DSo significa que no hay daño, DS1 daños leves, DS2 indica daños graves pero reparables y DS3 implica el reemplazo total del elemento. Observando la Fig. 4, si la aceleración de la primera planta alcanza un valor de $0.50 g$, hay un $21 \%$ de probabilidad de que no se produzcan daños, un $9 \%$ de la probabilidad de que se produzcan daños DS1, un $47 \%$ de que el daño sea DS2, y un $23 \%$ de que el estado de daños sea DS3. Los parámetros valor medio $(\lambda)$ y desviación estándar

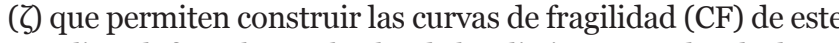
estudio y definen los umbrales de los distintos estados de daño, se han obtenido de $(2,37,38)$ y se resumen en la Tabla 3.

\subsection{Costes de reparación}

Los costes de reparación se estiman como una fracción del coste inicial del edificio. La estimación de los costes totales es 2,1M€, de forma que la repercusión por metro cuadrado es $1335 €$. En la Tabla 3 se resume el coste inicial de cada uno de los grupos de prestaciones. Los costes de reparación de los diferentes estados de daño DSo, 1, 2, y 3 son o \%, 23\% 66\% y

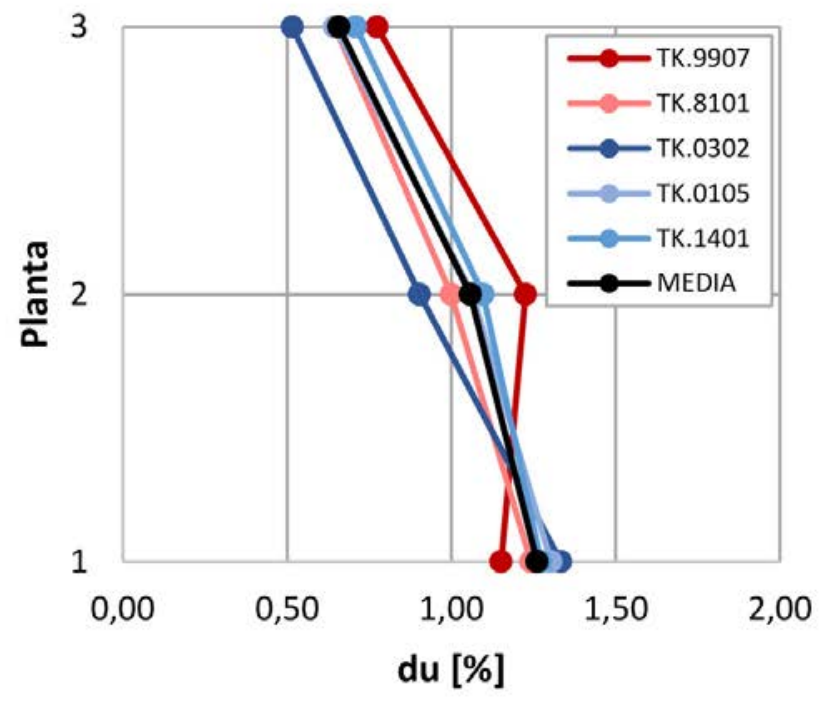

(a) Deriva entre plantas. Sismo raro.

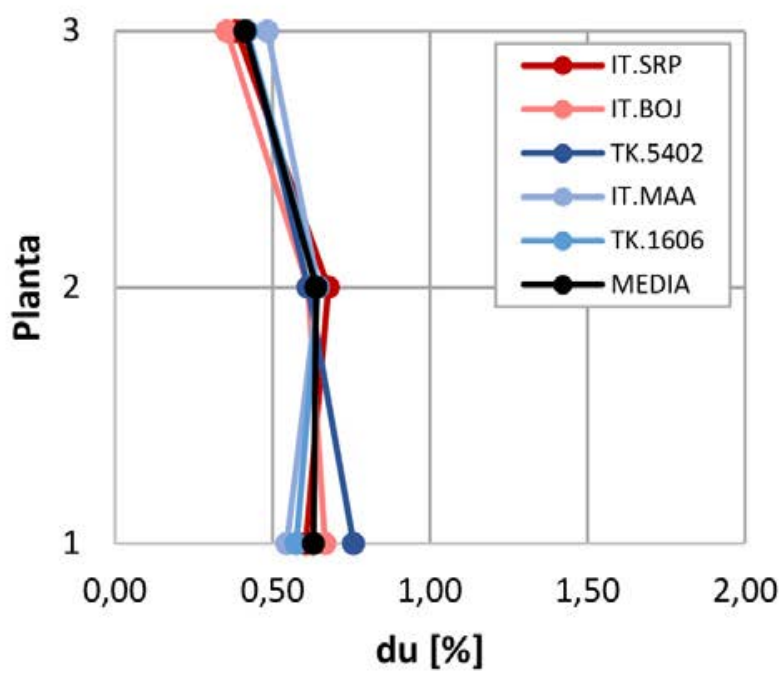

(c) Deriva entre plantas. Sismo ocasional.

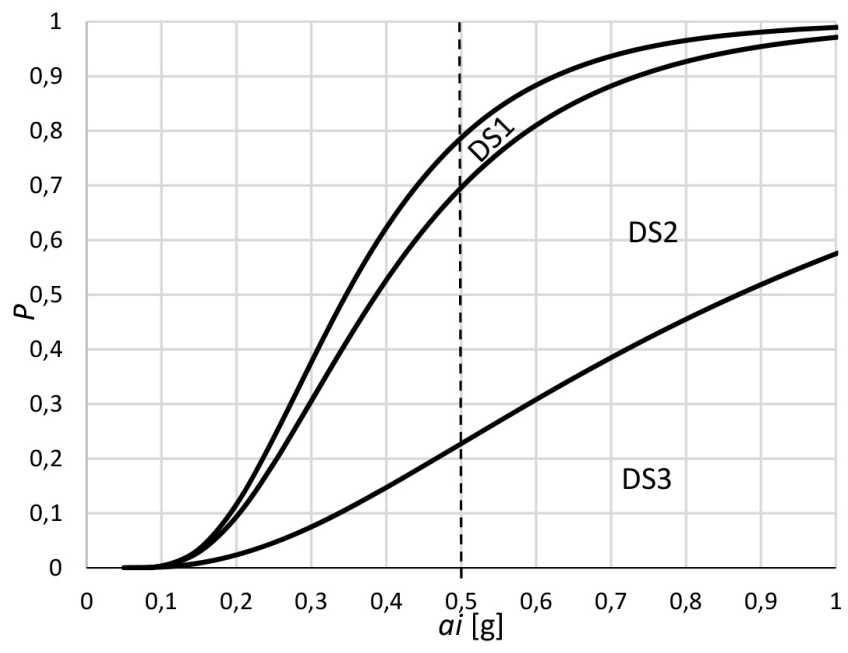

Figura 4. Curva de fragilidad para el GP58.

$100 \%$ del coste inicial del edificio. Si los grupos de prestaciones solo consideran los estados de daño $0,1,2$, los costes de reparación son $\mathrm{o} \%, 35 \%$ y $100 \%$ del coste inicial.

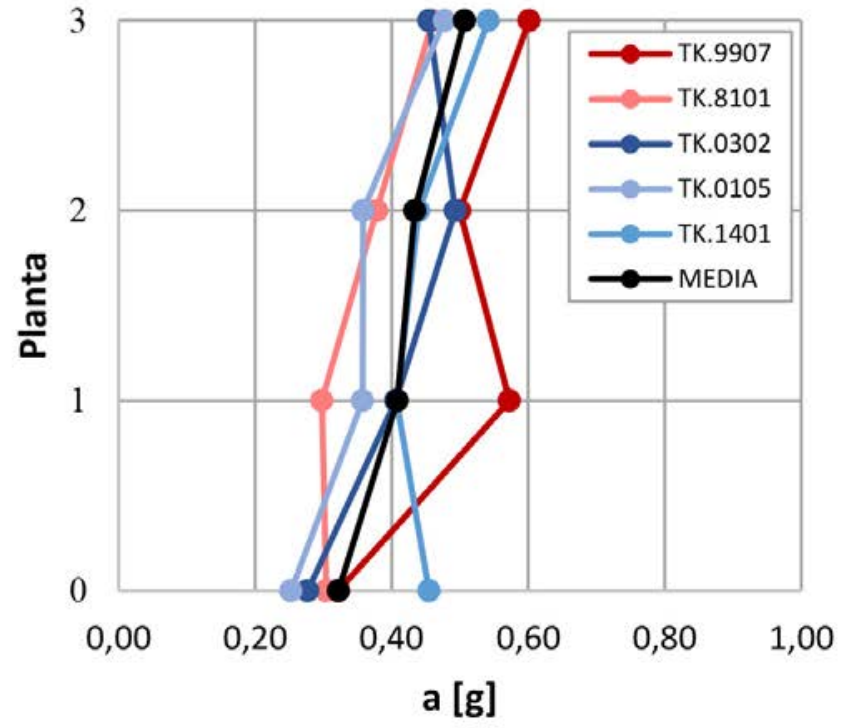

(b) Aceleración de planta. Sismo raro.

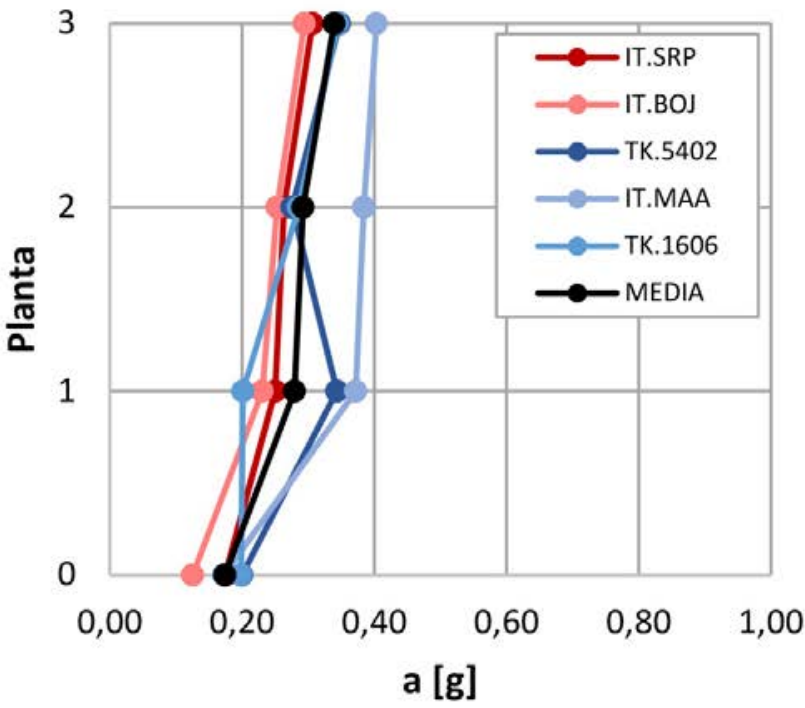

(d) Aceleración de planta. Sismo ocasional.

Figura 5. Parámetros de respuesta DP para sismos raro y ocasional. 


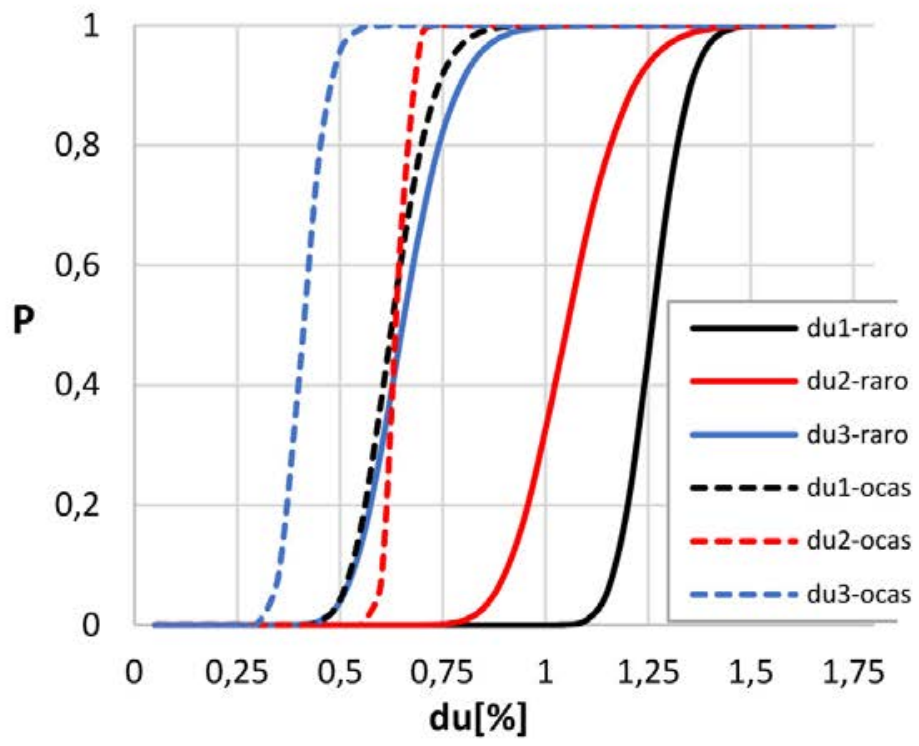

(a) Deriva entre plantas.

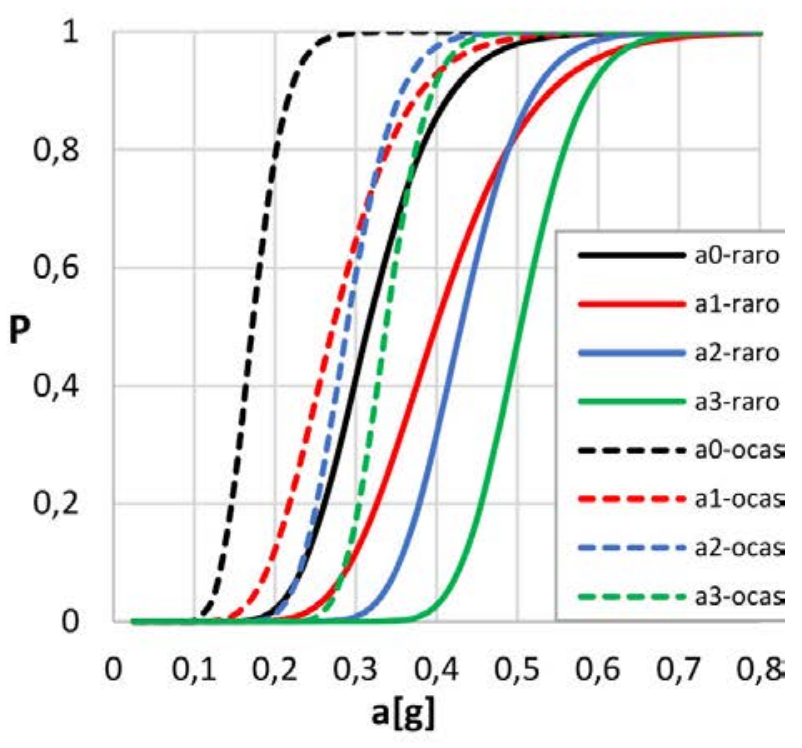

(b) Aceleración de planta.

Figura 6. Función de distribución acumulada de la respuesta estructural.

\section{ANÁLISIS DE LA RESPUESTA ESTRUCTURAL}

Los análisis dinámicos no lineales para el prototipo de edificio sanitario se llevan a cabo introduciendo en SeismoStruct (36) los cinco registros recopilados para cada una de las dos intensidades. Los dos parámetros obtenidos de los análisis son el desplazamiento pico entre plantas $d u_{i}$, expresado como porcentaje de la altura de planta, y la aceleración de planta $a_{i}$, recogidos en la Fig. 5 para sismos raros y ocasionales. El valor medio se muestra en líneas negras. Asumiendo que los valores de los DP siguen una distribución logarítmico-normal, la respuesta estructural puede expresarse con las funciones de distribución acumulada de la Fig. 6 para la deriva entre plantas y para la aceleración de planta.

Para terremotos raros, las medias de los valores del desplazamiento entre plantas están comprendidas entre el o,65\% y el $1,27 \%$ de la altura de planta, estando este último valor en el rango de los resultados experimentales obtenidos de ensayos realizados a pórticos de H.A. en mesa sísmica: 1.19\% (39). Los mayores desplazamientos se registran en planta baja, reduciéndose en pisos superiores. La aceleración media del suelo es $0,28 \mathrm{~g}$ y la aceleración horizontal de planta oscila entre $0,41 \mathrm{~g}$ y $0,50 \mathrm{~g}$.

Para terremotos ocasionales, las medias de los valores pico de la deriva entre plantas están entre $0,44 \%$ y o,61\%, en el rango del $0,5 \%$ observado en los experimentos de la referencia (39). La planta superior registra desplazamientos menores al $0,5 \%$, mientras que las plantas inferiores superan este valor en todos los casos observados. La aceleración media del suelo es $0,21 \mathrm{~g}$, y la aceleración horizontal media de planta varía entre $0,28 \mathrm{~g}$ y $0,36 \mathrm{~g}$.

\section{PRESTACIONES SÍSMICAS DEL EDIFICIO}

Los límites de desplome de planta empleados para evaluación de daños por la SEAOC (26) son 0,2- 0,5\% para ocupación inmediata (IO), 0,5-1,5\% para seguridad para las vidas (LS), y prevención del colapso (CP) 1,5-2,5\%. El desplome de planta para el estado de limitación de daño del Eurocódigo
8 (2) es similar al de ocupación inmediata del SEAOC: 0,5\% de la altura de planta si existen elementos frágiles anclados a la estructura. Ante el terremoto ocasional, podemos observar en la Fig. 6a que hay un $100 \%$ de probabilidades de que el límite de ocupación inmediata sea excedido en las dos primeras plantas del edificio, mientras que en la última planta no se supera. Esto implica que no se cumplen los requisitos de limitación del daño que establece el Eurocódigo 8 y que la respuesta del edificio ha alcanzado el nivel de prestaciones de seguridad para la vida, superando el nivel de prestaciones de ocupación inmediata.

Ante terremotos raros, todos los resultados están por debajo del 2,5\%, lejos del colapso estructural. Las prestaciones del edificio están en el rango de seguridad para las vidas, con daños extensos en variedad de elementos del edificio. Considerando que el edificio analizado es una instalación sanitaria y que se aplica en el proyecto un factor de sobrerresistencia, las prestaciones no son satisfactorias y el edificio no sigue totalmente operativo en ninguno de los dos escenarios planteados.

Siguiendo la metodología planteada, los niveles de daño en los elementos del edificio se estiman aplicando el teorema de la probabilidad total a partir de los valores de DP y las curvas de fragilidad. La Fig. 7 muestra los estados de daño (DS) resultantes para los grupos de prestaciones del edificio.

En el evento del terremoto de proyecto la Fig. 7a muestra que los grupos de prestaciones que se caracterizan por un estado de daños DS3, que implica el reemplazo del elemento son: la estructura, tabiquería, carpinterías, ascensores, y alumbrado de emergencia. Otros elementos arquitectónicos (cerramientos, falsos techos, petos, puertas) presentan estados de daño variados, pero es anecdótico que permanezcan sanos. En las instalaciones del edificio, el daño se concentra en las luminarias y luces de emergencia, con daños en sistemas de detección y alarma de incendios. En los contenidos, los daños más importantes ocurren en el equipamiento médico, con un predominio de los estados de daño DS2 y DS3. Se observan esporádicamente daños graves en equipos informáticos y el vuelco de estanterías. La usabilidad de esta instalación sani- 


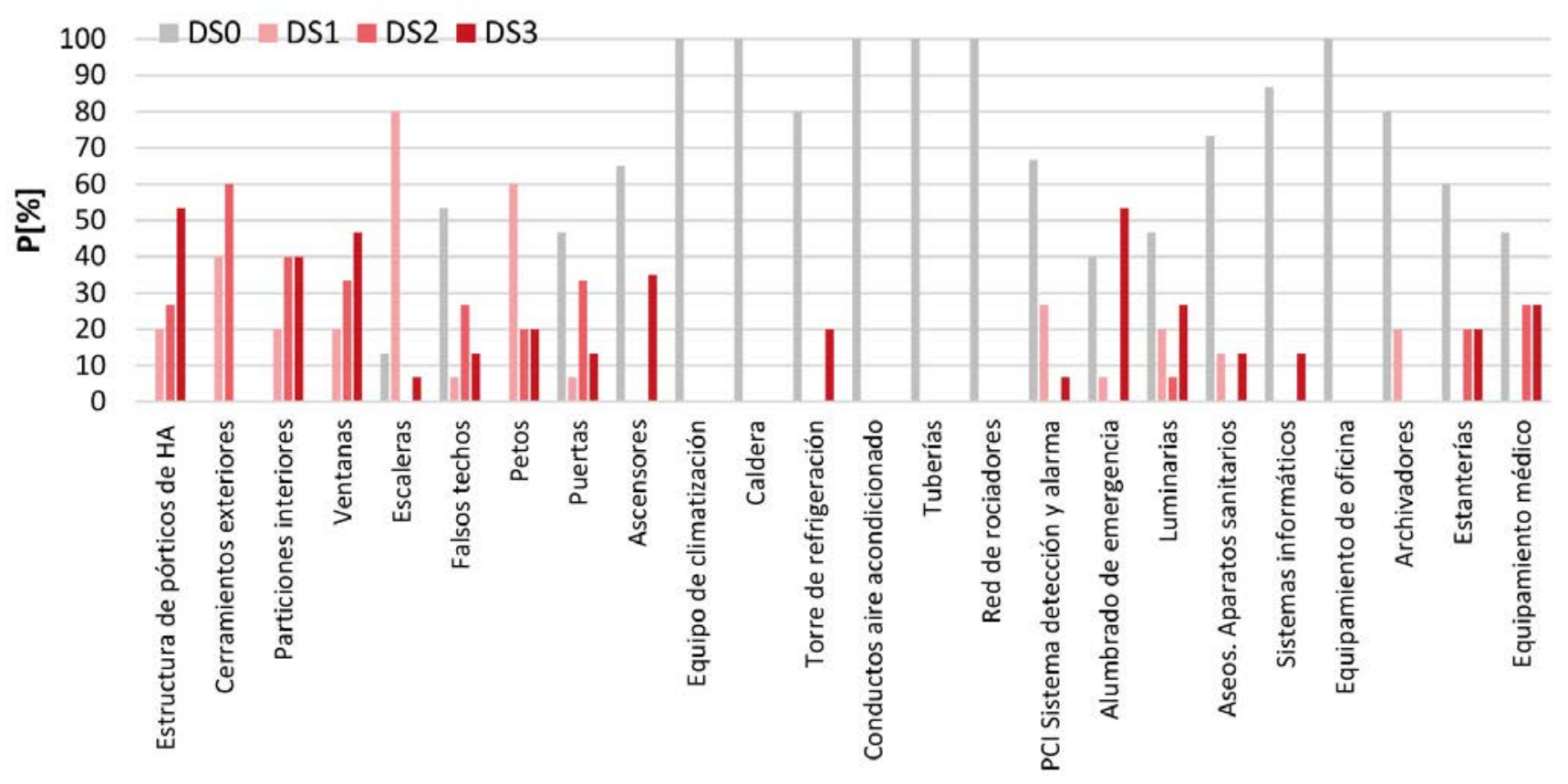

(a)

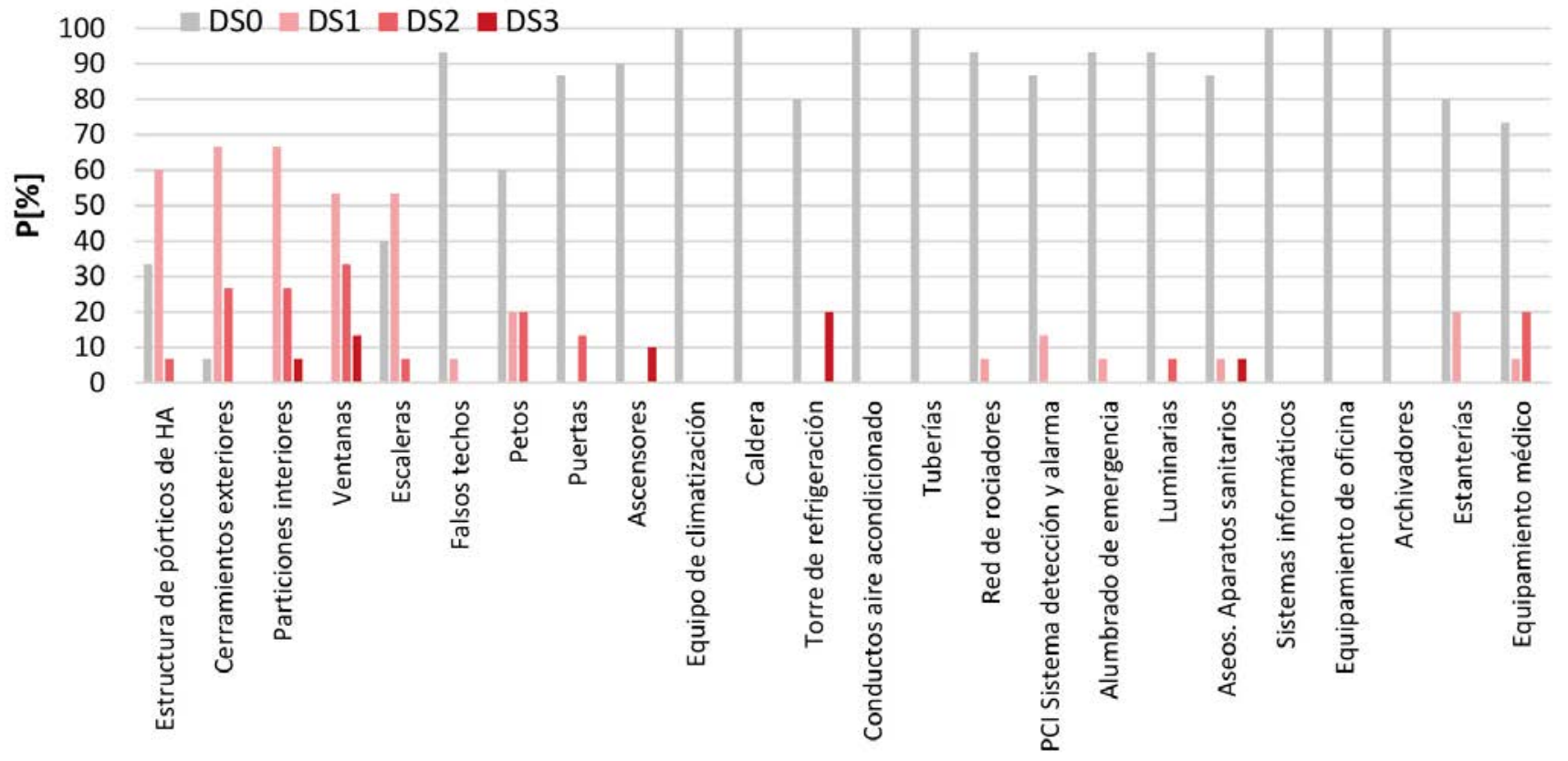

(b)

Figura 7. Estados de daños tras sismos raros (a) y ocasionales (b).

taria queda sin duda comprometida: ascensores y sistemas de climatización inoperativos, pérdidas de rigidez y capacidad lateral ante réplicas, necesidad de reparaciones y reemplazos, y equipamiento médico dañado. Las prestaciones del edificio son de seguridad para las vidas, ya que no es posible su ocupación inmediata. El comportamiento estructural está lejos del colapso, pero los daños a los elementos no estructurales (roturas de tabiques, falsos techos, petos, vidrios) presentan riesgos significativos durante la evacuación. En la Fig. 6b se muestra que la probabilidad de que la aceleración pico de planta primera y segunda sea superior a 0,5g es del $20 \%$. Las implicaciones en el equipamiento médico de que la aceleración pico oscile entre $0,5^{-1} \mathrm{~g}$ incluyen: oscilación de armarios, deslizamiento de escritorios o contenido de estanterías que caen al suelo y por tanto obstaculizan.
En el caso de terremotos ocasionales, los estados de daño en la Fig. 7b muestran daños no graves (DS1-2) en la estructura en el $67 \%$ de los casos, y los elementos arquitectónicos muestran daños entre leves y graves pero reparables en cerramientos y tabiques. La probabilidad de que aparezcan daños en cerramientos es del $93 \%$, y del 100\% en la tabiquería y ventanas. Las instalaciones del edificio se mantienen sin daños en más del $95 \%$ de los análisis, con daños graves puntualmente en elementos sensibles a la aceleración de la planta de cubierta, como luminarias, aparatos sanitarios o las torres de refrigeración. El equipamiento médico sufre daños en un $27 \%$ de los casos observados.

En términos de estado de servicio, el análisis de daños indica la interrupción de la actividad en ambos escenarios de inten- 
sidad. Para el terremoto ocasional, son esperados daños leves y graves en la mayor parte de los elementos constructivos del edificio y de forma especial, en los elementos sensibles al desplome de planta, como cerramientos, tabiquería, y carpinterías. Esto sugiere que la instalación quedaría fuera de servicio. La inspección y reparación de la estructura de hormigón armado y los elementos no estructurales conlleva un tiempo de inactividad.

La probabilidad de que los costes de reparación no excedan una determinada suma se muestra en las curvas de distribución acumulada de la Fig. 8 para ambas intensidades sísmicas, oscilando entre $0,12-0,75 \mathrm{M} €$ para terremotos ocasionales, $\mathrm{y}$ entre $0,5-1,25 \mathrm{M} €$ para terremotos raros. Esto representa entre el $6-36 \%$ y el $24-57 \%$ de los costes iniciales, respectivamente. Hay un $50 \%$ de probabilidades de que los respectivos costes de reparación estén sobre 0,25M€ y 0,77M€.

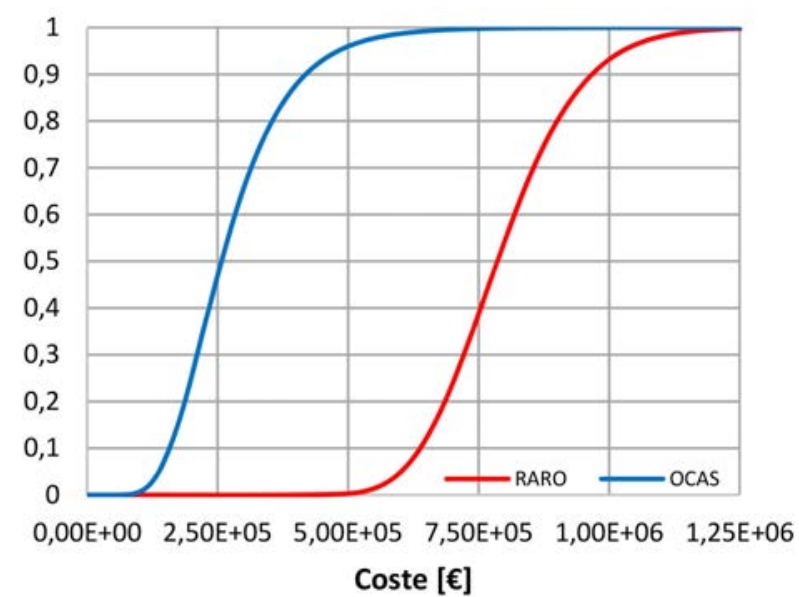

Figura 8. Función de distribución acumulada de los costes totales.

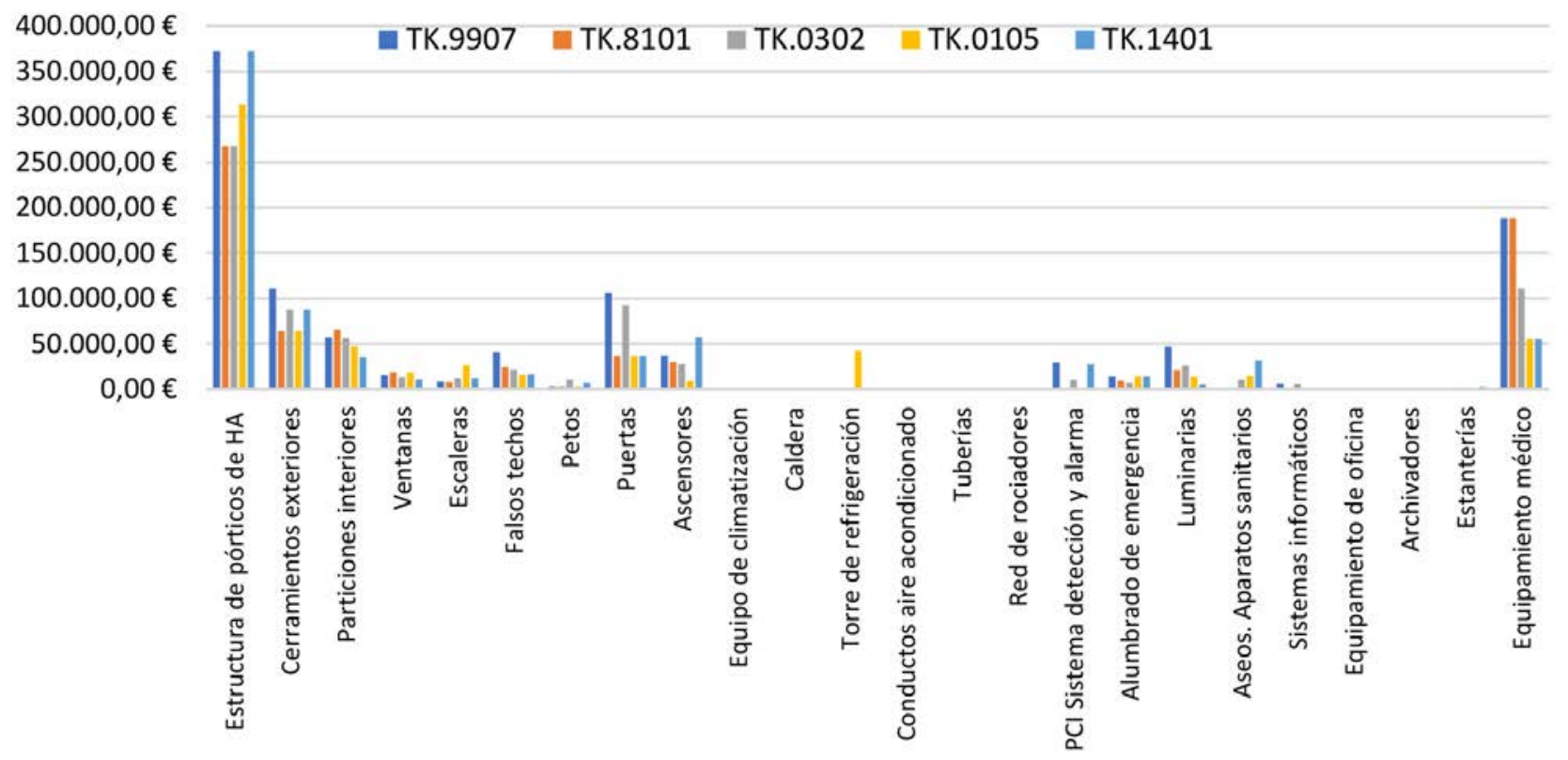

(a)

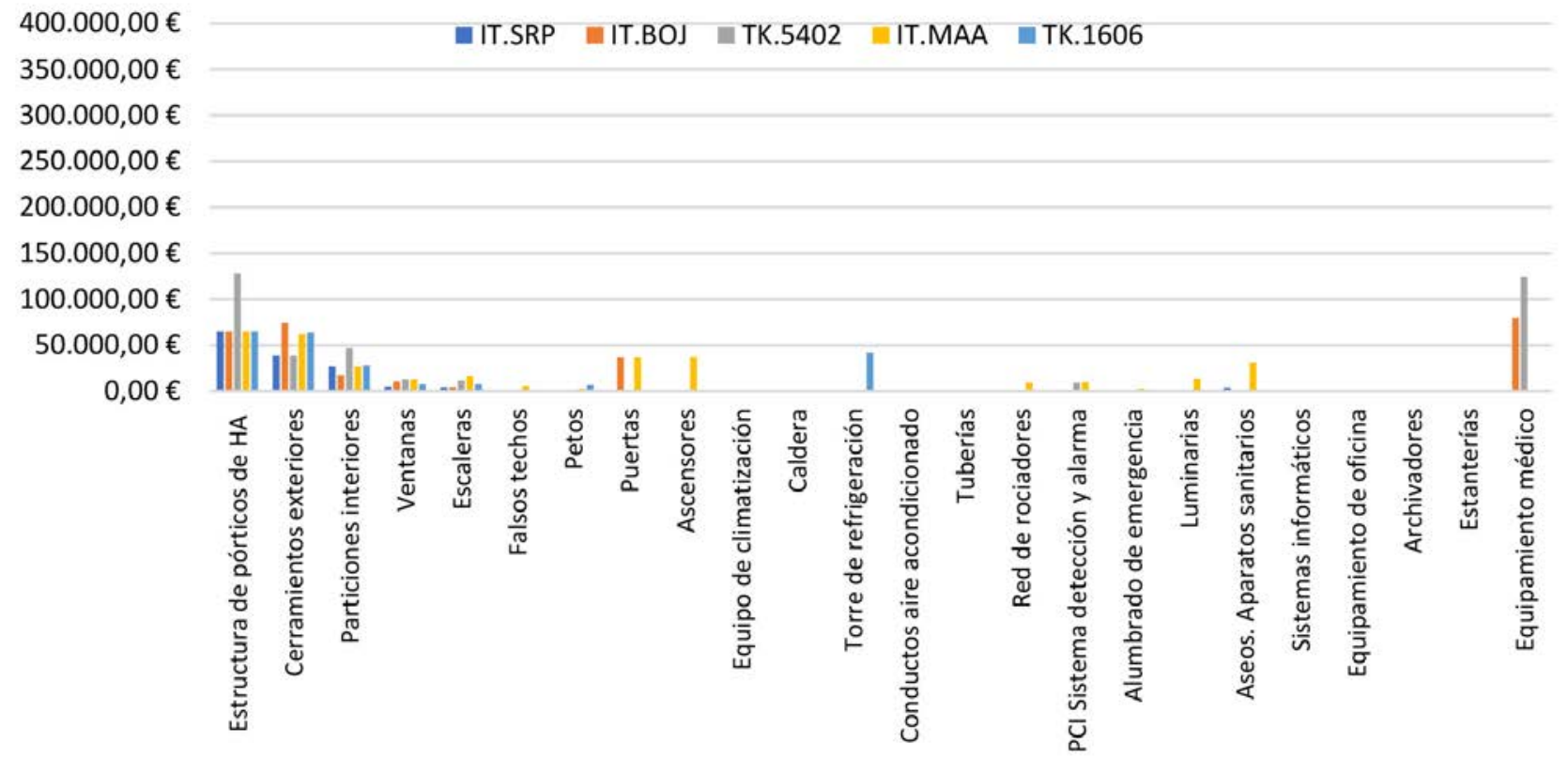

(b)

Figura 9. Costes de reparación para cada grupo de prestaciones. a) Sismo raro. b) Sismo ocasional. 
La Fig. 9 muestra los GP que representan los costes de reparación más elevados. Después de un terremoto raro, los costes más importantes en la Fig. 9a son atribuidos a los elementos estructurales, seguidos por la reparación de daños en elementos no estructurales como equipamiento médico, cerramientos, particiones, puertas, acristalamientos, ascensores, techos, luces, alarmas de incendios, luces de emergencia, equipamiento médico, aparatos sanitarios, torres de refrigeración e incluso sistemas informáticos. Los costes de reparación relacionados con un terremoto ocasional en la Fig. 9b son debidos en su mayor parte a los daños estructurales y a la reparación generalizada de daños en elementos no estructurales como cerramientos, particiones, ascensores, torres de refrigeración y también el equipamiento médico.

\section{CONCLUSIONES}

Se discuten las prestaciones sismorresistentes de un edificio sanitario en una zona de sismicidad moderada y proyectado con la normativa española. La estrategia de la NCSE-02 para edificios de importancia especial consiste en dar más resistencia lateral a la estructura ante el terremoto de proyecto, amplificando las fuerzas laterales por un factor de 1,3. En el caso de una instalación crítica, las prestaciones sismorresistentes ideales deben permitir la ocupación inmediata del edificio después de un terremoto ocasional, y garantizar la seguridad después de un sismo raro. Sin embargo, en base al análisis de la respuesta, daños, y costes, pueden observarse daños considerables que afectan seriamente al funcionamiento del edificio.

Después de un terremoto ocasional, es dudoso que la instalación pueda permanecer operativa debido a los daños generalizados en estructura y elementos arquitectónicos. Las recomendaciones que establece el Eurocódigo 8 para el estado de limitación de daños no se cumplen en dos tercios del edificio. El nivel de prestaciones resultante está entre la seguridad de la vida y la ocupación inmediata. La reparación de daños en elementos no estructurales representa un 55-80\% del coste total de reparación e implica que la instalación permanezca fuera de servicio un periodo de tiempo. Hay un $50 \%$ de probabilidades de que los costes de reparación alcancen el $11 \%$ del coste del edificio.

Después de un sismo raro, se observan daños severos en numerosos elementos estructurales y no estructurales, requiriendo su evaluación o reemplazo. La pérdida de rigidez y resistencia laterales pueden hacer que la instalación sea sensible a sucesivas réplicas. Los costes de reparación podrían representar el entre el 27 y $54 \%$ de los costes iniciales de construcción del edificio. El refuerzo de elementos estructurales representa el 52-65\% de los costes de reparación. Daños en puertas y fuertes movimientos de accesorios pueden bloquear las salidas de evacuación. Los niveles de prestaciones estarían comprendidos entre seguridad de la vida y prevención del colapso. Las aceleraciones de planta son especialmente perjudiciales para el equipamiento médico y sistemas informáticos, lo que puede alterar la asistencia sanitaria después del evento.

En base al estudio, es necesaria la correcta consideración de los estados de limitación de daños, para alcanzar resultados satisfactorios en términos de habitabilidad, mitigación de daños y controlar pérdidas económicas. La aplicación simultánea del factor de amplificación de fuerza y factores de reducción de resistencia impiden el entendimiento del potencial del terremoto. Las consecuencias de un sismo raro o de proyecto son extremas, y los efectos de los sismos ocasionales no son menos importantes. Por lo tanto, se requiere la evaluación basada en prestaciones y el refuerzo de edificios existentes para asegurar que permanezcan seguros y operativos después del evento. Es también necesario asignar una protección adecuada para los sistemas críticos y contenidos.

\section{REFERENCIAS}

(1) NCSE (2002). Norma de Construcción Sismorresistente: Parte General y Edificación. Ministerio de Fomento.

(2) Eurocódigo 8 (2012). UNE-EN 1998-3:2012 Proyecto de estructuras sismorresistente. Parte 3: Evaluación y adecuación sísmica de edificios. Asociación Española de Nomalización (AENOR).

(3) ASCE 7 (2010). Minimum design loads for buildings and other structures. American Society of Civil Engineers (ASCE).

(4) ATC-13 (1985). Earthquake damage evaluation data for California. Applied Technology Council, Redwood City.

(5) Ho, C., Hadj-Hamou, T., Nilsson, M. (1995, 17 de Octubre). GIS based zonation of infraestructural damage related to seismically triggered landslide risk. En Proceedings of the Fifth International Conference on Seismic Zonation (pp. 142149). Nantes: Ouest Editions.

(6) FEMA 577 (2010). Design Guide for Improving Hospital Safety in Earthquakes, Floods, and High Winds. Federal Emergency Management Agency.

(7) Çeleri, M. (1996). Comparison of Northridge earthquake response of a base-isolated and conventional hospital building. Proceedings of the 11th World Conference on Earthquake Engineering. Paper No. 25. Acapulco. México.

(8) Retamales, R., Mosqueda, G., Filiatrault, A., Reinhorn, A. (2011). Testing protocol for experimental seismic qualification of distributed nonstructural systems. Earthquake Spectra, 27(3): 835-856. doi: http://dx.doi.org/10.1193/1.3609868.

(9) Whittaker, A.S., Soong, T.T. (2003, 23-24 de Octubre). An overview of nonstructural component research at three U.S. earthquake engineering research centers. En Proceedings of Seminar on Seismic Design, Performance and Retrofit of Nonstructural Components in Critical Facilities. (pp 271-280). Los Ángeles: ATC-29-2.

(10) Filiatrault, A., Timothy, S. (2014). Performance-based seismic design of nonstructural building components: The next frontier of earthquake engineering. Earthquake Engineering and Engineering Vibration 13(1):17-46. doi: http://dx.doi. org/10.1007/s11803-014-0238-9.

(11) FEMA 461 (2007). Interim Testing Protocols for Determining the Seismic Performance Characteristics of Structural and Nonstructural Components. Federal Emergency Management Agency.

(12) ICC-ES AC156 (2006). Acceptance Criteria for Seismic Certification by Shake-Table Testing of Nonstructural Components. ICC Evaluation Service. 
(13) FEMA E-74 (2012). Reducing the Risks of Nonstructural Earthquake Damage. Federal Emergency Management Agency.

(14) BS ISO 13033:2013 (2013). Bases for design of structures. Loads, forces and other actions. Seismic actions on nonstructural components for building applications. Organización Internacional de Normalización (ISO).

(15) BS EN 81-77:2013 (2013). Safety rules for the construction and installations of lifts. Particular applications for passenger and goods passenger lifts. Lifts subject to seismic conditions. British Standards Institution.

(16) BS EN 60255-21-1:1996 (1996), IEC 60255-21-1:1988. Electrical relays. Vibration, shock, bump and seismic tests on measuring relays and protection equipment. Vibration tests (sinusoidal). British Standards Institution.

(17) BS EN 60255-21-2:1996 (1996), IEC 60255-21-2:1988. Electrical relays. Vibration, shock, bump and seismic tests on measuring relays and protection equipment. Shock and bump tests. British Standards Institution.

(18) Maffei, J., Bazzurro, P. (2004). The 2002 Molise, Italy, earthquake. Earthquake Spectra 20(S1): S1- S22;. doi: http:// dx.doi.org/10.1193/1.1770976.

(19) Price, H.J., De Sortis, A., Schotanus, M. (2012). Performance of the San Salvatore Regional Hospital in the 2009 L'Aquila Earthquake. Earthquake Spectra 28(1): 239-256. doi: http://dx.doi.org/10.1193/1.3673595.

(20) Masi, A., Santarsiero, M., Gallipoli, M., Mucciarelli, V., Manfredi, A., Dusi, A., Stabile, T.A. (2013) Performance of the health facilities during the 2012 Emilia (Italy) earthquake and analysis of the Mirandola hospital case study. Bulletin of Earthquake Engineering 12(5):2419-2443. doi: http://dx.doi.org/10.1007/s10518-013-9518-4.

(21) Cosenza, E., Di Sarno, L., Maddaloni, G., Magliulo, G., Petrone, C., Prota, A. (2015). Shake table tests for the seismic fragility evaluation of hospital rooms. Earthquake Engineering \& Structural Dynamics 44(1):23-40. doi: http://dx.doi. org/10.1002/eqe.2456.

(22) Sato, E., Furukawa, S., Kakehi, A., Nakashima, M. (2011). Full-scale shaking table test for examination of safety and functionality of base-isolated medical facilities. Earthquake Engineering \& Structural Dynamics 2011; 40(13): 14351453. doi: http://dx.doi.org/10.1002/eqe.1097.

(23) Shi, Y., Masahiro, K., Masayoshi, N. (2014). Disorder and damage of base-isolated medical facilities when subjected to near-fault and long-period ground motions. Earthquake Engineering \& Structural Dynamics 43(11): 1683-1701. doi: http://dx.doi.org/10.1002/eqe.2417.

(24) Kuo, K.C., Suzuki, Y., Katsuragi, S., Yao, G.C. (2011) Shake table test on clutter levels of typical medicine shelves and contents subjected to earthquakes. Earthquake Engineering \& Structural Dynamics 40(12): 1367-1386. doi: http:// dx.doi.org/10.1002/eqe.1094.

(25) Furukawa, S., Sato, E., Shi, Y., Becker, T., Nakashima, M. (2013). Full-scale shaking table test of a base-isolated medical facility subjected to vertical motions. Earthquake Engineering \& Structural Dynamics 42(13): 1931-1949. doi: http:// dx.doi.org/10.1002/eqe.2305.

(26) Structural Engineers Association of California \& Vision 2000 Committee. Performance Based Seismic Engineering of Buildings. California Office of Emergency Services: Sacramento, 1995

(27) ASCE/SEI 31-03 (2003). Seismic Evaluation of Existing Buildings. American Society of Civil Engineers (ASCE).

(28) ASCE/SEI 41-06 (2007). Seismic Rehabilitation of Existing Buildings. American Society of Civil Engineers (ASCE).

(29) ATC-58-1 (2011). Seismic Performance Assessment of Buildings - Volume 1 - Methodology. Applied Technology Council. Redwood City, California.

(30) Moehle, J., Deierlein, G.G. (2004, 1-6 de Agosto). A framework methodology for performance- based earthquake engineering. En 13th world conference on earthquake engineering: (pp. 3812-3814). Vancouver, Canadá.

(31) Abrahamson, N. A. (1992). Non-stationary spectral matching. Seismological research letters, 63(1), 30.

(32) Hancock, J., Watson-Lamprey, J., Abrahamson, N. A., Bommer, J. J., Markatis, A., McCoy, E. M. M. A., \& Mendis, R. (2006). An improved method of matching response spectra of recorded earthquake ground motion using wavelets. Journal of earthquake engineering, 10(speco1), 67-89. doi: http://dx.doi.org/10.1142/S1363246906002736.

(33) CTE (2006). Código Técnico de la Edificación. Ministerio de Vivienda.

(34) EHE-o8 (2008). Instrucción del Hormigón Estructural. Secretaría Técnica General. Ministerio de Fomento.

(35) CYPE Ingenieros SA (2012). CYPECAD 2012.k [Software]. Disponible en http://descargas.cype.es

(36) SeismoSoft (2016) SeismoStruct [Software] A computer program for static and dynamic nonlinear analysis of framed structures. Disponible en http://www.seismosoft.com.

(37) Yao, C., Tu, L.Y. (2012, 1-12 de Marzo). The generation of earthquake damage probability curves for building facilities in Taiwan. En Proceedings of the International Symposium on Engineering Lessons from the 2011 Great East Japan Earthquake. Tokyo, Japón.

(38) ATC-58 (2012). Performance Assessment Calculation Tool. Applied Technology Council: Redwood City, California.

(39) Benavent-Climent, A., Morillas, L., Escolano-Margarit, D. (2014). Shake-table tests of a reinforced concrete frame designed following modern codes: seismic performance and damage evaluation. Earthquake Engineering \& Structural Dynamics 43(6):791-810. doi: http://dx.doi.org/10.1002/eqe.2372. 